\title{
Identification of the Propeller Coefficients and Dynamic Parameters of a Hovering Quadrotor from Flight Data
}

\author{
Damien Six ${ }^{1}$, Sébastien Briot $^{2}$, Julian Erskine ${ }^{1}$ and Abdelhamid Chriette ${ }^{1}$
}

\begin{abstract}
Several methods can be applied to estimate the propeller thrust and torque coefficients and dynamics parameters of quadrotor UAVs. These parameters are necessary for many controllers that have been proposed for these vehicles. However, these methods require the use of specific test benches, which do not well simulate real flight conditions.

In this paper, a new method is introduced which allows the identification of the propeller coefficients and dynamic parameters of a quadrotor in a single procedure. It is based on a Total-Least-Square identification technique, does not require any specific test bench and needs only a measurement of the mass of the quadrotor and a recording of data from a flight that can be performed manually by an operator.

Because the symmetries of classic quadrotors limit the performance of the algorithm, an extension of the procedure is proposed. Two types of flights are then used: one with the initial quadrotor and a second flight with an additional payload on the vehicle that modifies the mass distribution. This new procedure, which is validated experimentally, increases the performance of the identification and allows an estimation of all the relevant dynamic parameters of the quadrotor near hovering conditions.
\end{abstract}

Index Terms-Aerial Systems: Mechanics and Control, Calibration and Identification

\section{INTRODUCTION}

Q UADROTOR UAVs have recently experienced increasing interest with advances in modeling, control and path planning. Many control techniques have been proposed for these vehicles [1]-[8]. Several of those control laws require a knowledge of the UAV's dynamics parameters (e.g. mass and inertia parameters) and propeller thrust and torque coefficients, and are sensitive to the accuracy of the parameters. An accurate estimation of the parameters increases the precision and robustness of the controllers and is therefore required.

Several methods can be applied to estimate the parameters of quadrotor-style UAVs:

Manuscript received: September 6, 2019; Revised December 5, 2019 Accepted December 30, 2019.

This paper was recommended for publication by Editor Jonathan Roberts upon evaluation of the Associate Editor and Reviewers' comments.

This work was supported by the RFI AtlanSTIC2020 project RAPID

${ }^{1}$ D. Six, J. Erskine and A. Chriette are with the Laboratoire des Sciences du Numérique de Nantes (LS2N), UMR CNRS 6004, at the École Centrale de Nantes, 1 Rue de la Noe, 44321, Nantes, France. \{Damien.Six, Julian.Erskine, Abdelhamid.Chriette als2n.fr

${ }^{2} \mathrm{~S}$. Briot is with the LS2N at the Centre National de la Recherche Scientifique, 44321, Nantes, France. Sebastien. Briotels2n.fr

Digital Object Identifier (DOI): see top of this page
- Estimation of the frame dynamic parameters from design [9], [10]: This method can lead to an unevaluated lack of precision in the estimation of the parameters.

- Experimental estimation of the structure parameters: Apart from the mass which is directly measured with a scale, the other parameters require specific test benches. As an example, in [11], the inertia parameters are measured indirectly with a pendulum device. With such methods, the precision of the inertia parameters are impacted by the precision of the other ones previously measured.

- Estimation of the dynamic parameters from the analysis of flight data [12]-[14] by means of a Kalman filter assuming the knowledge of, at least, the propeller coefficients of thrust and torque.

Those algorithms all require a knowledge of the propeller coefficients. The estimation of those coefficients and the modelling of aerodynamic effects are studied with specific test benches [15]-[18] and may not be completely equivalent to those appearing during a free flight of the drone.

For classical robotic manipulators, most of the dynamic offline identification methods use an Inverse Dynamic Identification Model (IDIM), that gives a linear relation between the joint forces/torques and the dynamic parameters, and estimate the parameter values using least squares techniques (LS) [19][21]. This procedure is called the IDIM-LS technique. An extension of this method using Total Least Square (IDIMTLS) is also used to identify dynamic parameters and joint drive gains of robot manipulators in a single procedure [22].

In this research, a new method is proposed to identify all the structure and rotor parameters for a quadrotor UAV, inspired by the IDIM-TLS procedure. Our approach aims to obtain an identification of the dynamic parameters of the device without any specific test bench or a computation of some of the parameters from design, as other methods would generally require. It uses the command reference of the propeller velocities, and the flight data collected while the robot is operated by a human pilot. All the dynamic parameters and propeller coefficients are calculated in one step as the Total Least Square solution (TLS) of an overdetermined system. The method only requires a knowledge of the vehicle mass, which is easily measured using a scale. This algorithm was established in the scope of a project on aerial manipulation. Thus, the study is limited to near hovering flight configurations.

The paper is organized as follows. In the next Section, we define the inverse dynamic identification model of the quadrotor. In Section III] the TLS identification procedure is 


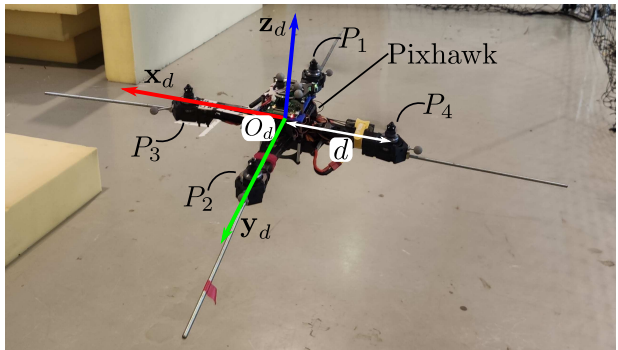

Fig. 1. Local frame and propeller positions $P_{1} \ldots P_{4}$ of the quadrotor.

introduced. Two different types of data can be collected in order to feed the identification model: data from the Inertial Measurement Unit (IMU) or from a motion capture system (MOCAP). Identification results by using the first or second set of data are then compared in the Section IV] Because a classic quadrotor presents some symmetries that will lead to a failure of the identification of some dynamic parameters using the TLS method, an extended version of the procedure is introduced in order to overcome this limitation. It is based on a modification of the mass distribution of the UAV and recordings of two types of flight data: a first set for the usual quadrotor, and second set with a modified configuration of the quadrotor. Finally, in Section V, conclusions are drawn.

\section{DYNAMIC MODEL OF A QUADROTOR}

The quadrotor vehicle consists of four individual rotors attached to a rigid cross airframe (Fig. 11). The control of a quadrotor is achieved by differential control of the thrust and drag torques generated by each rotor. The system is underactuated, with only four inputs available to control the six Degrees of Freedom (DoF). The dynamics of the quadrotor are described through a combination of the rigid body dynamic equations (Section II-A) and the model of the propellers (Section II-B).

\section{A. Rigid body dynamics}

Let us consider a mobile frame $\mathcal{F}_{d}\left(O_{d}, \mathbf{x}_{d}, \mathbf{y}_{d}, \mathbf{z}_{d}\right)$ attached to the quadrotor (Fig. 1). The origin of this frame $O_{d}$ is at the middle of the cross. The $\mathbf{x}_{d}$ axis is placed along the axis of one of the arms and the $\mathbf{z}_{d}$ axis is aligned with the quadrotor propellers axes. A standard assumption in quadrotor modelling and control is to consider this origin as the Center of Mass (CoM) of the vehicle. However, this assumption is not guaranteed in general. We aim to identify the real position of the CoM with the identification procedure. For this purpose, a general model of the rigid body dynamics is used. In this model, mechanical effects affecting the main body of the quadrotor are described. This study however neglects aerodynamical forces acting on the quadrotor frame. Those forces become non negligible at high speed (over $5 \mathrm{~m} / \mathrm{s}$ ) [23] but those flight configurations are out of the scope of this study. The ten dynamic parameters of a rigid body are the followings:

- The mass $m$
- The 3 components $m s_{x}, m s_{y}, m s_{z}$ of the first moment of inertia ms in the local frame, at its origin $O_{d}$,

- The 6 components of the symmetric inertia matrix in the local frame, at its origin $O_{d}$,

$$
\mathbf{I}=\left[\begin{array}{lll}
I_{x x} & I_{x y} & I_{x z} \\
I_{x y} & I_{y y} & I_{y z} \\
I_{x z} & I_{y z} & I_{z z}
\end{array}\right]
$$

The mass is the only parameter that is easily measured. All the others unknown parameters are regrouped in a vector $\chi$ :

$$
\chi^{T}=\left[m s_{x}, m s_{y}, m s_{z}, I_{x x}, I_{y y}, I_{z z}, I_{x y}, I_{x z}, I_{y z}\right]
$$

The following notations are also defined

- $\mathbf{g}=[0,0,-g]^{T}, g>0$, is the gravity vector, defined in the world frame.

- $\boldsymbol{\zeta}$ is the position of the mobile frame origin $O_{d}$.

- $\mathbf{a}_{d}=\left[a_{x}, a_{y}, a_{z}\right]^{T}$ is the acceleration of $O_{d}$ expressed in the local frame $\mathcal{F}_{d}$.

- $\mathbf{R}$ is the rotation matrix defining the orientation of the mobile frame with respect to the world frame. In this paper, the rotation sequence of the Bryant angles $\left(\boldsymbol{\eta}=[\psi, \theta, \phi]^{T}\right)$ is chosen, i.e. $\mathbf{R}=\mathbf{R}_{\mathbf{z}}(\psi) \mathbf{R}_{\mathbf{y}}(\theta) \mathbf{R}_{\mathbf{x}}(\phi)$. $\mathbf{R}_{\mathbf{u}}(\alpha)$ is the elemental rotation about axis $\mathbf{u}$ though the angle $\alpha$.

- $\boldsymbol{\omega}=\left[\omega_{x}, \omega_{y}, \omega_{z}\right]^{T}$ is the angular velocity of the mobile frame, expressed in frame $\mathcal{F}_{d}$.

- $\mathbf{f}, \boldsymbol{\tau}$ are the vectors of forces and the torques applied to the quadrotor by the propellers at $O_{d}$, expressed in $\mathcal{F}_{d}$.

The Newton-Euler equations, expressed at the body frame origin $O_{d}$ in body frame $\mathcal{F}_{d}$ are given in [21] as:

$$
\begin{aligned}
& m \mathbf{a}_{d}+\dot{\boldsymbol{\omega}} \times \mathbf{m s}+\boldsymbol{\omega} \times(\boldsymbol{\omega} \times \mathbf{m s})=m \mathbf{R}^{-1} \mathbf{g}+\mathbf{f} \\
& \mathbf{I} \dot{\boldsymbol{\omega}}+\boldsymbol{\omega} \times \mathbf{I} \boldsymbol{\omega}+\mathbf{m s} \times \mathbf{a}_{d}=\mathbf{m s} \times\left(\mathbf{R}^{-1} \mathbf{g}\right)+\boldsymbol{\tau}
\end{aligned}
$$

Equations (1) and (2) give the dynamics of the quadrotor body as functions of the measured variables usually available from the embedded IMU. In this paper, we consider also measurements from a MOCAP system. Those measurements are the position of $O_{d}$ given in world frame $\zeta$ and the orientation coordinates given in Bryant angles $\boldsymbol{\eta}$. The acceleration $\mathbf{a}_{d}$ in the body frame is related to $\boldsymbol{\eta}$ through

$$
\mathbf{a}_{d}=\mathbf{R}^{-1} \ddot{\boldsymbol{\zeta}}
$$

The angular velocity $\boldsymbol{\omega}$ and its time derivative are related to the first and second time derivatives of Bryant angles $\boldsymbol{\eta}$ by

$$
\begin{aligned}
& \boldsymbol{\omega}=\mathbf{T}_{\eta} \dot{\boldsymbol{\eta}} \\
& \dot{\boldsymbol{\omega}}=\dot{\mathbf{T}}_{\eta} \dot{\boldsymbol{\eta}}+\mathbf{T}_{\eta} \ddot{\boldsymbol{\eta}}
\end{aligned}
$$

The matrix $\mathbf{T}_{\eta}$ depends on the rotation representation chosen [24]. Using (3) and (4), the dynamic equations (1) and (2) as function of the measurements of the MOCAP and their derivatives are given by

$$
\begin{aligned}
m \mathbf{R}^{-1} \ddot{\boldsymbol{\zeta}} & +\left(\dot{\mathbf{T}}_{\eta} \dot{\boldsymbol{\eta}}+\mathbf{T}_{\eta} \ddot{\boldsymbol{\eta}}\right) \times \mathbf{m s} \\
& +\mathbf{T}_{\eta} \dot{\boldsymbol{\eta}} \times\left(\mathbf{T}_{\eta} \dot{\boldsymbol{\eta}} \times \mathbf{m s}\right)=m \mathbf{R}^{-1} \mathbf{g}+\mathbf{f} \\
\mathbf{I}\left(\dot{\mathbf{T}}_{\eta} \dot{\boldsymbol{\eta}}\right. & \left.+\mathbf{T}_{\eta} \ddot{\boldsymbol{\eta}}\right)+\mathbf{T}_{\eta} \dot{\boldsymbol{\eta}} \times \mathbf{I} \mathbf{T}_{\eta} \dot{\boldsymbol{\eta}}+\mathbf{m s} \times \mathbf{R}^{-1} \ddot{\boldsymbol{\zeta}} \\
& =\mathbf{m s} \times\left(\mathbf{R}^{-1} \mathbf{g}\right)+\boldsymbol{\tau}
\end{aligned}
$$




\section{B. Propellers model}

The steady-state wrench provided by a hovering propellor in free air along the $\mathbf{z}_{d}$ axis is composed of a thrust $\mathbf{f}_{p}$ and a drag torque $\boldsymbol{\tau}_{p}$ given by [2]

$$
\mathbf{f}_{p}=k_{t} \Omega^{2} \mathbf{z}_{d}, \quad \boldsymbol{\tau}_{p}=-\operatorname{sign}(\Omega) k_{d} \Omega^{2} \mathbf{z}_{d}
$$

The $\operatorname{sign}(x)$ function extracts the sign of a real number $x . \Omega$ is the propeller rotational velocity about $\mathbf{z}_{d}$. In the scope of this study (near hovering), $k_{t}$ and $k_{d}$ are considered as positive coefficients named respectively thrust and torque coefficients. They depend on the rotor design and are grouped in a vector named $\chi_{k}$, i.e. $\chi_{k}^{T}=\left[k_{t} k_{d}\right]$. In a real flight, there are many aerodynamic (blade flapping, ground and ceiling effects, etc) [25] and gyroscopic effects associated with any rotor craft that modify the simple force model introduced above. However, those effects are generally neglected or considered as disturbances for control application [26] [2] and will not be considered in our model.

Assuming that the thrust and torque coefficients are the same for all the propellers, the contribution of all the propellers are summed and expressed at the frame origin $O_{d}$. The total wrench $\mathbf{w}=\left[\begin{array}{ll}\mathbf{f}^{T} & \boldsymbol{\tau}^{T}\end{array}\right]^{T}$ acting on the body (see Fig. 1 for the position of each propeller) is

$$
\mathbf{f}=\left[\begin{array}{c}
0 \\
0 \\
k_{t} \sum_{i} \Omega_{i}^{2}
\end{array}\right], \boldsymbol{\tau}=\left[\begin{array}{c}
k_{t} d\left(-\Omega_{1}^{2}+\Omega_{2}^{2}\right) \\
k_{t} d\left(-\Omega_{3}^{2}+\Omega_{4}^{2}\right) \\
k_{d} \sum_{i}-\operatorname{sign}\left(\Omega_{i}\right) \Omega_{i}^{2}
\end{array}\right]
$$

where

- $d$ is the distance of a propeller axis from $O_{d}$ along $\mathbf{x}_{d}$ and $\mathbf{y}_{d}$.

- $\Omega_{i}$ is the angular velocity of the propeller $i$ around $\mathbf{z}_{d}$.

The dynamic model of the hovering quadrotor is obtained through the equations of its body dynamics (1)-(2) and the equation of the total wrench applied by the propellers 9 . For other multi-rotors, this method only requires a modification of (9) to include the wrench effects of the extra propellers.

\section{Inverse Dynamic Identification Model (IDIM)}

The IDIM-TLS procedure [22] requires an expression of the dynamic model linear w.r.t. the dynamic parameters and propeller coefficients that need to be identified. This linearity is well known for classical robots [21] and can be easily obtained for the quadrotor dynamic model.

Equations (17, (2) and (9) giving the dynamic model of a quadrotor are linear w.r.t. the mass $m$, the unknown dynamic parameters $\chi$ and the coefficients of the rotors grouped in $\chi_{k}$. Then the dynamic model can be written under the form

$$
m \mathbf{D}_{m}+\mathbf{D} \boldsymbol{\chi}=\mathbf{D}_{k} \boldsymbol{\chi}_{k}
$$

with

$$
\begin{aligned}
& \mathbf{D}=\left[\begin{array}{ccccc}
-\omega_{y}^{2}-\omega_{z}^{2} & \omega_{x} \omega_{y}-\dot{\omega}_{z} & \omega_{x} \omega_{z}+\dot{\omega}_{y} & 0 & 0 \\
\omega_{x} \omega_{y}+\dot{\omega}_{z} & -\omega_{x}^{2}-\omega_{z}^{2} & -\dot{\omega}_{x}+\omega_{y} \omega_{z} & 0 & 0 \\
\omega_{x} \omega_{z}-\dot{\omega}_{y} & \dot{\omega}_{x}+\omega_{y} \omega_{z} & -\omega_{x}^{2}-\omega_{y}^{2} & 0 & 0 \\
0 & a_{z}+g c \phi c \theta & -a_{y}-g s \phi c \theta & \dot{\omega}_{x} & -\omega_{y} \omega_{z} \\
-a_{z}-g c \phi c \theta & 0 & a_{x}-g s \theta & \omega_{x} \omega_{z} & \dot{\omega}_{y} \\
a_{y}+g s \phi c \theta & -a_{x}+g s \theta & 0 & -\omega_{x} \omega_{y} & \omega_{x} \omega_{y}
\end{array}\right. \\
& \left.\begin{array}{cccc}
0 & 0 & 0 & 0 \\
0 & 0 & 0 & 0 \\
0 & 0 & 0 & 0 \\
\omega_{y} \omega_{z} & -\omega_{x} \omega_{z}+\dot{\omega}_{y} & \omega_{x} \omega_{y}+\dot{\omega}_{z} & \omega_{y}^{2}-\omega_{z}^{2} \\
-\omega_{x} \omega_{z} & \dot{\omega}_{x}+\omega_{y} \omega_{z} & -\omega_{x}^{2}+\omega_{z}^{2} & -\omega_{x} \omega_{y}+\dot{\omega}_{z} \\
\dot{\omega}_{z} & \omega_{x}^{2}-\omega_{y}^{2} & \dot{\omega}_{x}-\omega_{y} \omega_{z} & \omega_{x} \omega_{z}+\dot{\omega}_{y}
\end{array}\right] \\
& \mathbf{D}_{k}^{T}=\left[\begin{array}{cc}
0 & 0 \\
0 & 0 \\
\sum_{i} \Omega_{i}^{2} & 0 \\
d\left(-\Omega_{1}^{2}+\Omega_{2}^{2}\right) & 0 \\
d\left(-\Omega_{3}^{2}+\Omega_{4}^{2}\right) & 0 \\
0 & \sum_{i}-\operatorname{sign}\left(\Omega_{i}\right) \Omega_{i}^{2}
\end{array}\right] \\
& \mathbf{D}_{m}=\left[\begin{array}{llllll}
a_{x}-g s \theta & a_{y}+g s \phi c \theta & a_{z}+g c \phi c \theta & 0 & 0 & 0
\end{array}\right]^{T}
\end{aligned}
$$

where $c$ and $s$ are shorthand for cosinus and sinus.

Because of perturbations due to measurement noise and modeling errors, the model (10) differs from reality by an error $\mathbf{e}$ such that

$$
m \mathbf{D}_{m}+\mathbf{D} \boldsymbol{\chi}=\mathbf{D}_{k} \boldsymbol{\chi}_{k}+\mathbf{e}
$$

Equation (11) is called the Inverse Dynamic Identification Model (IDIM). Note that the IDIM model may be extended to include any additional effects as long as the dynamic model remains linear w.r.t. the coefficients of those effects. Non linear coefficients have to be identified either with a separate study or with a linear approximation.

\section{IDENTIFICATION OF THE QUADROTOR DYNAMIC PARAMETERS USING THE TOTAL LEAST SQUARE (TLS) PROCEDURE}

\section{A. Identification of the propellers coefficients and dynamic parameters}

The off-line identification of the UAV's dynamic parameters can be achieved thanks to the data obtained for feeding the matrices $\mathbf{D}_{m}, \mathbf{D}$ and $\mathbf{D}_{k}$. The data are collected while the vehicle is flying (methodology is discussed in Section [III-C). The model (11) is sampled, low-pass filtered and, in standard identification procedures like [20] decimated in order to get the following overdetermined linear system of $n \times 6$ equations and 11 unknowns (variables in $\chi$ and $\chi_{k}$ ):

$$
\mathbf{W}_{k} \boldsymbol{\chi}_{k}+\mathbf{W} \boldsymbol{\chi}+m \mathbf{W}_{m}=\boldsymbol{\rho}
$$

in which:

- $\mathbf{W}=\left[\begin{array}{llll}\mathbf{D}_{1}^{T} & \mathbf{D}_{2}^{T} & \ldots & \mathbf{D}_{n}^{T}\end{array}\right]^{T}$,

- $\mathbf{W}_{m}=\left[\begin{array}{llll}\mathbf{D}_{m 1}^{T} & \mathbf{D}_{m 2}^{T} & \ldots & \mathbf{D}_{m n}^{T}\end{array}\right]^{T}$,

- $\mathbf{W}_{k}=-\left[\begin{array}{llll}\mathbf{D}_{k 1}^{T} & \mathbf{D}_{k 2}^{T} & \ldots & \mathbf{D}_{k n}^{T}\end{array}\right]^{T}$,

- $\boldsymbol{\rho}=\left[\begin{array}{llll}\mathbf{e}_{1}^{T} & \mathbf{e}_{2}^{T} & \ldots & \mathbf{e}_{n}^{T}\end{array}\right]^{T}$,

where $\mathbf{D}_{i}=\mathbf{D}\left(t=t_{i}\right), \mathbf{D}_{m i}=\mathbf{D}_{m}\left(t=t_{i}\right)$ and $\mathbf{D}_{k i}=$ $\mathbf{D}_{k}\left(t=t_{i}\right)(i=1, \ldots, n)$ are the matrices $\mathbf{D}, \mathbf{D}_{m}$ and $\mathbf{D}_{k}$, respectively, computed with the motion's data collected at time $t=t_{i}$, while $\mathbf{e}_{i}$ is the error of the model at time $t_{i}$. 
Considering that the mass $m$ of the quadrotor is accurately known, and grouping all unknowns $\chi$ and $\chi_{k}$ into a single vector $\chi_{t}, 12$ is re-expressed as

$$
\mathbf{W}_{t} \chi_{t}=\rho
$$

where $\mathbf{W}_{t}=\left[\mathbf{W}_{k} \mathbf{W} m \mathbf{W}_{m}\right]$ is a $(r \times 12)(r=6 n)$ matrix and $\chi_{t}=\left[\begin{array}{lll}\chi_{k}^{T} & \chi^{T} & 1\end{array}\right]^{T}$ is a vector of dimension 12 .

Based on this form of equation, it is possible to find the unknowns in $\chi_{t}$ thanks to a Total Least Square identification procedure [27], [28]. Indeed, without perturbation, $\boldsymbol{\rho}=\mathbf{0}$. As a result, $\mathbf{W}_{t}$ should be rank deficient. Then would exist an infinite number of solutions to the Eq. (13) that are all in the kernel of $\mathbf{W}_{t}$ :

$$
\hat{\chi}_{t}=\lambda \hat{\chi}_{t}^{*}
$$

where $\hat{\chi}_{t}^{*}$ is the unit vector such that $\mathbf{W}_{t} \hat{\chi}_{t}^{*}=\mathbf{0}$ and $\lambda$ is a scalar value.

Due to measurement perturbations and, in our case, model errors, $\mathbf{W}_{t}$ is of full rank. Therefore the system $(13)$ is changed to the closest compatible system w.r.t. the Frobenius norm. This system is obtained through the "thin" Singular Value Decomposition (SVD) of $\mathbf{W}_{t}$.

$$
\mathbf{W}_{t}=\mathbf{U S V}^{T}
$$

where $\mathbf{U}$ and $\mathbf{V}$ are $\left(r \times n_{t}\right)\left(n_{t}=12\right)$ and $\left(n_{t} \times n_{t}\right)$ matrices and $\mathbf{S}$ is a diagonal matrix with the singular values $s_{j}$ of $\mathbf{W}_{t}$ sorted in decreasing order. The closest rank deficient matrix $\overline{\mathbf{W}}_{t}$ is given by

$$
\overline{\mathbf{W}}_{t}=\mathbf{W}_{t}-s_{n_{t}} \mathbf{U}_{n_{t}} \mathbf{V}_{n_{t}}^{T}
$$

where $s_{n_{t}}$ is the smallest singular value of $\mathbf{W}_{t}$ and $\mathbf{U}_{n_{t}}$ (respectively $\mathbf{V}_{n_{t}}$ ) is the last column of $\mathbf{U}$ (respectively $\mathbf{V}$ ). Then, the normalized solution $\hat{\chi}_{t}^{*}$ such that

$$
\overline{\mathbf{W}}_{t} \hat{\chi}_{t}^{*}=\mathbf{0}
$$

is given by the last column of $\mathbf{V}$, i.e. $\hat{\chi}_{t}^{*}=\mathbf{V}_{n_{t}}$.

There is an infinite number of solutions $\hat{\chi}_{t}=\lambda \hat{\chi}_{t}^{*}$ to equation (17). However, the only solution respecting the second condition given by (13), i.e. last value of $\hat{\chi}_{t}$ equal to one, is obtained for $\lambda=1 / \chi_{n_{t}}$, where $\chi_{n_{t}}$ is the last value of $\hat{\chi}_{t}^{*}$. Then, the dynamic parameters identified are given by $\hat{\chi}_{t}=\hat{\chi}_{t}^{*} / \chi_{n_{t}}$.

\section{B. Statistical analysis, essential parameters and weighted TLS}

In order to compute the standard deviations $\sigma_{\hat{\chi}_{j}}(j=$ $1, \ldots, 11)$ on the dynamic and propeller coefficients, it is assumed that all errors in data matrix $\mathbf{W}_{t}$ are independently and identically distributed with zero mean, and the common covariance matrix $\mathbf{C}_{W W}$ is defined by

$$
\mathbf{C}_{W W}=\hat{\sigma}_{W}^{2} \mathbf{I}_{r n_{t}}
$$

where $\mathbf{I}_{r n_{t}}$ is the identity matrix of dimension $\left(r n_{t} \times r n_{t}\right)$. In [27], an unbiased estimation of the standard deviation $\hat{\sigma}_{W}$ is provided:

$$
\hat{\sigma}_{W}=s_{n_{t}} / \sqrt{r-n_{t}}
$$

In [27], the covariance matrix of the estimation error is also approximated by

$$
\mathbf{C}_{\hat{\chi} \hat{\chi}} \simeq \sigma_{W}^{2}\left(1+\left\|\hat{\chi}_{1: n-1}\right\|_{2}^{2}\right)\left(\overline{\mathbf{W}}_{t_{1: n_{t}-1}^{T}}^{T} \overline{\mathbf{W}}_{t_{1: n_{t}-1}}\right)^{-1}
$$

with $\hat{\chi}_{1: n_{t}-1}$ the vector containing the $n_{t}-1$ first coefficients of $\hat{\chi}$ and $\overline{\mathbf{W}}_{t_{1: n_{t}-1}}$ a matrix composed of the $n_{t}-1$ first columns of $\overline{\mathbf{W}}_{t}$. Then the standard deviation of the $j$ th parameter is given by

$$
\sigma_{\hat{\chi}_{j}}=\sqrt{\mathbf{C}_{\hat{\chi} \hat{\chi}}(j, j)}
$$

with $\mathbf{C}_{\hat{\chi} \hat{\chi}}(j, j)$ the $j$ th diagonal coefficient of $\mathbf{C}_{\hat{\chi} \hat{\chi}}$. The relative standard deviation of the $j$ th parameter is given by

$$
\% \sigma_{\hat{\chi}_{j}}=100 \sigma_{\hat{\chi}_{j}} /\left|\hat{\chi}_{j}\right|
$$

During the identification process, some small parameters remain poorly identifiable because they have no significant contribution in the dynamic model. They can be canceled in order to simplify the dynamic model and to improve the quality of identification of the other parameters. These parameters are called "essential" parameters and are calculated using an iterative procedure starting from the initial parameters estimation. At each step the parameter which has the largest relative standard deviation is canceled. A new TLS parameter estimation of the simplified model is carried out. The procedure ends when $\max \left(\% \sigma_{\hat{\chi}_{j}}\right) / \min \left(\% \sigma_{\hat{\chi}_{j}}\right)<r_{\sigma}$, where $r_{\sigma}$ is a ratio defined by the user. The remaining essential parameters are enough to describe the dynamic model of the quadrotor. Also, in order to improve the estimation of $\mathbf{W}_{t}$, the rows of $\mathbf{W}_{t}$ are weighted taking into account the confidence in the measurements. As proposed in [22], to improve the TLS solution, the following procedure is applied

1) The rows of $\mathbf{W}_{t}$ are regrouped as function for each component of the wrench $\mathbf{w}$ in $(9): \mathbf{W}_{t}=\left[\mathbf{W}_{1}^{T} \ldots \mathbf{W}_{6}^{T}\right]^{T}$ with $\mathbf{W}_{j}(j=1 \ldots 6)$ grouping all equations corresponding to the $j$ th component of $\mathbf{w}$.

2) Each submatrix $\mathbf{W}_{j}(j=1 \ldots 6)$ is weighted by the inverse of the standard deviation corresponding to the equations in $\mathbf{W}_{j}$.

\section{Data collection for the observation matrix}

The collection of data to fill the observation matrices can be provided by several measurement sources. For this paper, the propeller velocities $\Omega_{i}(i=1 \ldots 4)$ required for the observation matrix $\mathbf{W}_{k}$ are computed from the recordings of each motor speed command related to the Pulse Width Modulation command set in the electronic speed controller by an empirical law. The propeller dynamics are neglected in this study. If available, a direct measure of the propeller velocity will increase the quality of the identification procedure. Two sources are then explored to obtain data in order to estimate the observation matrices $\mathbf{W}_{m}$ and $\mathbf{W}$ : the drone IMU and an external MOCAP system. For classic robot identification techniques, the IMU is generally not a good source of data to conduct an identification process, as it is quite noisy and leads to drift biases when reconstructing the position. However, in the specific case of quadrotors, the local dynamic equations 
do not depend on the position of the device. In addition, the orientation only impacts the gravity force which is included in the accelerometer measurement. Thus it makes the IMU a very good candidate to collect data for the identification of the dynamic model of a quadrotor. As a result, we tested two types of data collection for $\mathbf{W}_{m}$ and $\mathbf{W}$ :

1) Data collection from IMU: The angular velocity $\omega$ and the acceleration (including gravity) $\mathbf{a}_{d}-\mathbf{R}^{-1} \mathbf{g}$ are measured with the gyroscope and accelerometer, respectively. Gyroscope biases are estimated and compensated by an online Extended Kalman Filter. The angular acceleration estimation $\hat{\dot{\omega}}$ is obtained by sampling, band-pass filtering the measure of $\boldsymbol{\omega}$ with zero-phase noncausal Butterworth filter and central difference algorithm [29]. Those three vectors are enough to compute the matrices $\mathbf{D}_{m}$ and $\mathbf{D}$ in $(10)$.

2) Data collection from MOCAP system: The position $\zeta$ and the orientation coordinates given in Bryant angles $\boldsymbol{\eta}$ are measured from the MOCAP system. Their derivatives $\hat{\dot{\zeta}}, \hat{\ddot{\zeta}}, \hat{\dot{\eta}}$ and $\hat{\ddot{\eta}}$ are obtained by sampling, band-pass filtering with zero-phase noncausal Butterworth filter and central difference algorithm. Then, the acceleration $\mathbf{a}_{d}$, the angular velocity and acceleration $\boldsymbol{\omega}, \dot{\omega}$ are computed respectively from (3) and (4).

Using the IMU as a data source has several advantages. It doesn't require any external sensor in addition to those usually available on a quadrotor. If the data collection is done onboard, there is also no data synchronization required as the IMU and the rotor speed data are both available on the UAV. Also the data collected from the MOCAP must be differentiated twice, while the acceleration is directly collected on the IMU. We would expect a better data quality from the IMU, however, depending on the IMU, its calibration and online measurement processing, the data may be quite noisy and/or biased. This is why we propose a comparison of the two data collection methods in this paper.

\section{CASE STUdY}

\section{A. Experimental protocol}

Experimental validation was performed using a custom quadrotor based on a Lynxmotion Crazy2fly frame, with MT2208 brushless DC motors, 12A ESCs, and plastic 8045 dual-blade propellers. The controller was a Pixhawk 2.4 running PX4-v1.7.3. Motor speeds and IMU data were gathered from the Pixhawk using an onboard Raspberry Pi 3B+. The pose of the UAV was gathered using a Qualisys MOCAP system with eight cameras. All data was nominally gathered at $200 \mathrm{~Hz}$.

In order to carry out robot dynamic identification, it is usual to make the robot move along "exciting" trajectories, i.e. optimized reference trajectories that can be computed by nonlinear minimization of a criterion function of the condition number of the observation matrix [30]. However, agressive maneuvers in automatic flight mode would require a fine tuned control strategy relying on an external system (such as the MOCAP system). The objective of this paper is to propose a method that does not require specific equipment and so the

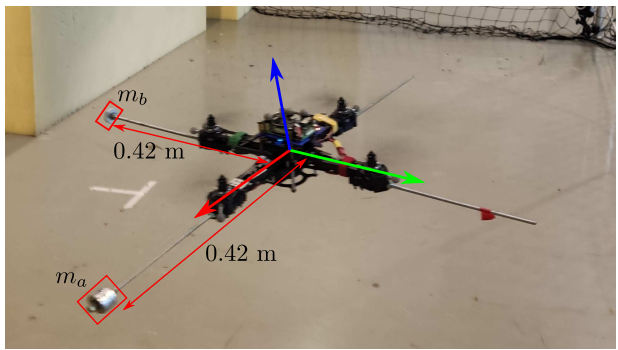

Fig. 2. Test configuration $\mathrm{B}$, were $m_{a}=0.125 \mathrm{~kg}$ and $m_{b}=0.042 \mathrm{~kg}$

trajectories were performed by a human pilot. Flights of 60 to 90 seconds were performed with a fully charged $11.1 \mathrm{~V}$ LiPo battery in a $6 \times 4 \times 4 \mathrm{~m}$ flying arena which limited the dynamics of the flights. While an effort was made to excite all dynamics of the quadrotor, the thrust and roll had the most variation while the pitch and yaw were less aggressive due to piloting skill and flight area constraints.

Tests were performed using two different quadrotor configurations. Configuration A corresponds to the base quadrotor with a measured mass of $1.285 \mathrm{~kg}$ (Fig. 11). For configuration $\mathrm{B}$, a $0.125 \mathrm{~kg}$ mass was added to the arm in the $+\mathrm{x}$ direction and a $0.042 \mathrm{~kg}$ mass to the arm in the $-\mathrm{y}$ direction (Fig. 2).

While an effort was made to acquire accurate data, the setup has several systematic flaws which decreased the quality of the experiment. A delay of at least $5 \mathrm{~ms}$ was present between the MOCAP data and motors. The motor velocity used here is the output reference for the speed controllers which have a non-zero rise time, and thus leads the actual motor speed by an unknown but likely non-negligible amount [9]. Another source of error is the rigid-body model of the quadrotor, when in reality it flexes. This particularly affects the roll-pitch dynamics of the drone where part of the actuation moments are used to flex the arms.

\section{B. Identification from IMU}

This section presents the identification results obtained from the IMU data. Inspired by the results obtained with two different configurations, we propose a procedure to identify a more complete set of dynamic parameter on a quadrotor. In order to distinguish the parameters associated to each configuration, parameters, vectors and matrices are annotated with the superscripts ${ }^{A}, B$ or ${ }^{A B}$ respectively if they concern the configuration A, B or both (resp.). The propeller coefficients are not associated to superscripts as they do not depend on the configuration of the drone.

The results obtained from the MOCAP data are presented and discussed in the next Section.

1) Configuration A: The first identification procedure, using the methodology presented in Section III is run on a quadrotor without additional payload (configuration A). The measured mass of the system is $1.285 \mathrm{~kg}$. The left-hand side of Table I presents the dynamic parameters identified with the procedure. The algorithm shows a very good performance for the identification of the thrust coefficient $k_{t}$ in comparison to the other parameters. This performance is required to set 
TABLE I

ESSENTIAL PARAMETERS IDENTIFIED FROM IMU DATA (SI BASE UNITS)

\begin{tabular}{l|cc}
\multicolumn{3}{c}{ Configuration A } \\
Param. & Value & $\% \sigma_{\hat{\chi}_{j}}$ \\
\hline${ }^{A} m_{s x}$ & $-4.32 \mathrm{E}-03$ & 2.70 \\
${ }^{A} m_{s y}$ & $-1.95 \mathrm{E}-03$ & 6.88 \\
${ }^{A} m_{s z}$ & $-6.16 \mathrm{E}-03$ & 4.55 \\
${ }^{A} I_{x x}$ & $1.69 \mathrm{E}-02$ & 1.52 \\
${ }^{A} I_{y y}$ & $1.76 \mathrm{E}-02$ & 1.45 \\
$k_{t}$ & $3.46 \mathrm{E}-06$ & 0.05
\end{tabular}

\begin{tabular}{l|cc}
\multicolumn{3}{c}{ Configuration B } \\
Param. & Value & $\% \sigma_{\hat{\chi}_{j}}$ \\
\hline${ }^{B} m_{s x}$ & $4.30 \mathrm{E}-02$ & 0.31 \\
${ }^{B} m_{s y}$ & $-2.01 \mathrm{E}-02$ & 0.80 \\
${ }^{B} m_{s z}$ & $-1.22 \mathrm{E}-02$ & 4.20 \\
${ }^{B} I_{x x}$ & $2.43 \mathrm{E}-02$ & 1.95 \\
${ }^{B} I_{y y}$ & $3.58 \mathrm{E}-02$ & 1.63 \\
${ }^{B} I_{z z}$ & $4.75 \mathrm{E}-02$ & 7.36 \\
$k_{t}$ & $3.65 \mathrm{E}-06$ & 0.04 \\
$k_{d}$ & $5.40 \mathrm{E}-08$ & 7.68
\end{tabular}

the ratio $r_{\sigma}$ (for computing the essential parameters, see Section III-B to 250 , so to avoid discarding too many parameters while selecting the essential ones (see also III-B).

The algorithm discards the dynamic parameters ${ }^{A} I_{x y},{ }^{A} I_{y z}$ and ${ }^{A} I_{x z}$. This result is expected as the mobile frame is aligned with the principal axes of inertia of the quadrotor. We can also see that the procedure discards the parameters $k_{d}$ and ${ }^{A} I_{z z}$. Those parameters are mainly related to the moment along the local $\mathbf{z}_{d}$ axis. To understand why those parameters are discarded, we can have a closer look at the equation related to this moment. Using the Table II we can assume the hypothesis: ${ }^{A} m_{s x} \simeq 0,{ }^{A} m_{s y} \simeq 0,{ }^{A} I_{x x} \simeq{ }^{A} I_{y y}$ and ${ }^{A} I_{x y}={ }^{A} I_{y z}={ }^{A} I_{x z}=0$. Under those hypotheses, from (10), we get

$$
{ }^{A} I_{z z} \dot{\omega}_{z} \simeq k_{d} \sum_{i}-\operatorname{sign}\left(\Omega_{i}\right) \Omega_{i}^{2}
$$

This equation is independent from all the other dynamic parameters. In addition, the term $k_{d}$ is not present in the other equations of the dynamic model and the ${ }^{A} I_{z z}$ terms are linearly related to ${ }^{A} I_{x x}$ or ${ }^{A} I_{y y}$ in the other equations of the drone's moments. This configuration makes the terms $k_{d}$ and ${ }^{A} I_{z z}$ completely independent from the other dynamic parameter and, thus, impossible to identify, knowing only the mass of the quadrotor. Note that $I_{z z}$ is often approximated as $I_{x x}+I_{y y}$ for quadrotors due to symmetry, but it is not seen as rigorous within the scope of this paper.

In order to identify the parameters associated with the moment about $\mathbf{z}_{d}$, it is necessary to break the symmetry of the quadrotor. Configuration B fills this requirement and will be used in a second identification procedure.

2) Configuration $B$ : The second identification procedure is run on a quadrotor with an additional payload that breaks the symmetry of the device (configuration B). The measured mass of the system is $1.452 \mathrm{~kg}$. The right-hand side of Table I presents the dynamic parameters identified with our procedure. Since the dynamic symmetry of the quadrotor is broken, the equation of the moment along $\mathbf{z}_{d}$ contains now other dynamic parameters in addition to the terms ${ }^{B} I_{z z}$ and $k_{d}$. This allows the algorithm to compute all the dynamic parameters, apart from the non diagonal terms of the inertia matrix which still do not sufficiently impact the dynamics of the quadrotor.

Since we obtained the torque coefficient $k_{d}$ with the configuration $\mathrm{B}$, it would be interesting to feed it in the computation of the configuration A to have a more complete identification of the initial quadrotor (which is the one we want to control
TABLE II

ESSENTIAL PARAMETERS ESTIMATED FROM COMBINED IDENTIFICATION PROCEDURE (SI BASE UNITS)

\begin{tabular}{l|cc||cc||c} 
& \multicolumn{2}{|c}{ IMU Data } & \multicolumn{2}{c}{ MOCAP Data } \\
Param. & Value & $\% \sigma_{\hat{\chi}_{j}}$ & Value & $\% \sigma_{\hat{\chi}_{j}}$ & $\% \delta$ \\
\hline${ }^{A} m_{s x}$ & $-4.54 \mathrm{E}-03$ & 3.35 & - & - & - \\
${ }^{A} m_{s z}$ & $-8.31 \mathrm{E}-03$ & 5.62 & - & - & - \\
${ }^{A} I_{x x}$ & $1.82 \mathrm{E}-02$ & 1.84 & $1.88 \mathrm{E}-02$ & 3.49 & 3.3 \\
${ }^{A} I_{y y}$ & $1.86 \mathrm{E}-02$ & 1.79 & $1.93 \mathrm{E}-02$ & 2.77 & 3.6 \\
${ }^{A} I_{z z}$ & $2.88 \mathrm{E}-02$ & 4.90 & - & - & - \\
${ }^{B} m_{s x}$ & $4.28 \mathrm{E}-02$ & 0.18 & $4.25 \mathrm{E}-02$ & 0.15 & 0.7 \\
${ }^{B} m_{s y}$ & $-1.99 \mathrm{E}-02$ & 0.47 & $-1.97 \mathrm{E}-02$ & 0.37 & 1.0 \\
${ }^{B} m_{s z}$ & $-1.30 \mathrm{E}-02$ & 3.20 & $-1.20 \mathrm{E}-02$ & 3.31 & 7.7 \\
${ }^{B} I_{x x}$ & $2.42 \mathrm{E}-02$ & 1.11 & $2.42 \mathrm{E}-02$ & 0.88 & 0.0 \\
${ }^{B} I_{y y}$ & $3.57 \mathrm{E}-02$ & 0.93 & $3.84 \mathrm{E}-02$ & 0.74 & 7.0 \\
${ }^{B} I_{z z}$ & $4.76 \mathrm{E}-02$ & 3.71 & - & - & - \\
$k_{t}$ & $3.63 \mathrm{E}-06$ & 0.03 & $3.68 \mathrm{E}-06$ & 0.02 & 1.4 \\
$k_{d}$ & $5.11 \mathrm{E}-08$ & 3.84 & - & - & -
\end{tabular}

$\%_{\delta}$ is the relative difference (in \%) between the values identified from IMU data and those identified from MOCAP data.

at the end), as the propellers coefficients are not modified from one configuration to another. We could have done that in two steps, getting the propellers coefficients from the identification of configuration $\mathrm{B}$ then use them as known variable in the identification of configuration A. However, computing in cascade the coefficients and then the inertia is not an efficient methodology because the error of identification of the coefficients will impact the identification of the inertia. As a result, in the next section, we propose an observation matrix that includes both configurations and allows the computation of all the dynamic parameters in a single computation.

3) Combined identification: The combined identification consist in solving both identifications for configurations A and $\mathrm{B}$ in a single TLS algorithm. The vector of the unknown

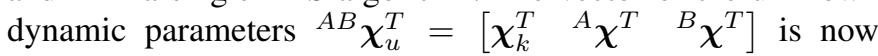
composed of 20 dynamic parameters: 9 inertia parameters associated to each configuration (A and $\mathrm{B}$ ) and 2 parameters for the propellers coefficients which are not dependent on the configuration. The observation matrix ${ }^{A B} \mathbf{W}_{t}$ used in the TLS equation is thus:

$$
{ }^{A B} \mathbf{W}_{t}{ }^{A B} \boldsymbol{\chi}_{t}=\boldsymbol{\rho},{ }^{A B} \boldsymbol{\chi}_{t}^{T}=\left[\begin{array}{ll}
\boldsymbol{\chi}_{u}^{T} & 1
\end{array}\right]
$$

It is equivalent to Eq. (13) and is then composed of several observation matrices as described by the following equation

$$
{ }^{A B} \mathbf{W}_{t}=\left[\begin{array}{cccc}
{ }^{A} \mathbf{W}_{k} & { }^{A} \mathbf{W} & \mathbf{0} & { }^{A} m^{A} \mathbf{W}_{m} \\
{ }^{B} \mathbf{W}_{k} & \mathbf{0} & { }^{B} \mathbf{W} & { }^{B} m^{B} \mathbf{W}_{m}
\end{array}\right]
$$

In order to represent the higher trust we have in the identification of configuration $\mathrm{B}$, an additional weighting was performed on the lines of the matrix ${ }^{A B} \mathbf{W}_{t}$ associated to the configuration B by a coefficient $k_{w}>1$ (in this case $k_{w}=2$ ).

The Table II presents the dynamic parameters identified with the procedure. As desired, the algorithm takes advantage of configuration $\mathrm{B}$ to estimate the parameter $k_{d}$ with the consequence of also allowing the estimation of the parameter ${ }^{A} I_{z z}$ for configuration A.

4) Cross-validation: In order to confirm the relevance of the identified parameters, a cross-validation is performed on flight data different from those used in the identification procedure. Figures 3 and 4 give the estimations obtained using 
TABLE III

RELATIVE ERROR NORMS $\|\boldsymbol{\rho}\| /\left\|\mathbf{W}_{k} \boldsymbol{\chi}_{k}\right\|(\%)$

\begin{tabular}{c|cccc}
\multicolumn{7}{c}{ Configuration A } \\
Wrench component & $F_{z}$ & $M_{x}$ & $M_{y}$ & $M_{z}$ \\
\hline \multicolumn{6}{c}{ Configuration B } & 43.73 & - \\
Wrench component & $F_{z}$ & $M_{x}$ & $M_{y}$ & $M_{z}$ \\
\hline \multicolumn{7}{c}{} & 4.40 & 20.99 & 10.70 & 45.19 \\
Configuration A & - Combined identification \\
Wrench component & $F_{z}$ & $M_{x}$ & $M_{y}$ & $M_{z}$ \\
\hline & 8.47 & 47.07 & 42.96 & 68.13 \\
Configuration B B Combined identification \\
Wrench component & $F_{z}$ & $M_{x}$ & $M_{y}$ & $M_{z}$ \\
\hline & 3.96 & 20.96 & 10.59 & 46.79
\end{tabular}

the identified parameters from the combined identification (Section IV-B3). Table III] gives the error norm relative to the motor inputs $\left(\|\boldsymbol{\rho}\| /\left\|\mathbf{W}_{k} \boldsymbol{\chi}_{k}\right\|\right)$ respectively for configurations $\mathrm{A}$ and $\mathrm{B}$ for all the identification procedures with the flight data for cross-validation. We can see that the estimations are completely relevant for the thrust. The moments are quite low in amplitude, so unmodelled effects can have a non-negligible impact on the accuracy of the estimations.

These unmodelled effects are several in nature: sensor biases and calibration, experimental recording delays, transitions states, body flexibility, aerodynamic effects, battery load, etc. Our estimation method gives the dynamic parameters for the imperfect dynamic model provided which is confronted with real flight data. It leads to the identification of dynamic parameters that may not be the real ones but those best fitted to the dynamic model used. The more precise the model, the closer to the real dynamic parameters the estimation will be. Nevertheless, in practice, the model provided should be the same for the identification and for the control law, because the identified values for a given model will be those leading to less modelling errors, and thus the best dynamic behavior.

\section{Identification from MOCAP}

Identification from MOCAP data was performed. However, worse data quality allowed the identification of fewer parameters, even with the combined identification procedure. The Table [I] shows the identified values for the dynamic parameters with data collected from the MOCAP system and a comparison to the data obtained with the IMU. The relative difference up to $7.7 \%$ is quite low considering that the reference frame between the two sensor systems are not perfectly aligned and that each system has its own calibration.

\section{CONCLusions}

In this paper we proposed a method to identify in one single procedure the rigid body parameters and thrust and torque coefficients of a hovering quadrotor from flight data. The procedure does not require any specific benchmark, and requires only the mass of the quadrotor and a recording of flight data (motor inputs and IMU outputs). The flight did not require a specific trajectory and can be performed manually by a somewhat skilled human operator as long as it remains "sufficiently exciting" [31] for the dynamics to be identified. The identification was performed in real flight conditions, giving the best fitting values in presence of perturbations and avoiding biases that may appear on a specific test bench.

We observed that the symmetries of a classic quadrotor limited the performance of the algorithm and we proposed an extended identification procedure which required two types of flights: one with the the initial quadrotor and a second flight with an additional payload on the vehicle that broke the mass distribution symmetries of the UAV. This new procedure increased the performance of the identification and allowed an estimation of all relevant dynamic parameters of the quadrotor.

The procedure has been presented in a near hovering context. Future work includes an extension of the procedure to aerodynamical effects in high speed flight. We believe also that it could be adapted to other multi-rotor UAVs.

\section{REFERENCES}

[1] D. Mellinger, N. Michael, and V. Kumar, "Trajectory generation and control for precise aggressive maneuvers with quadrotors," The International Journal of Robotics Research, vol. 31, no. 5, pp. 664-674, 2012.

[2] R. Mahony, V. Kumar, and P. Corke, "Multirotor Aerial Vehicles: Modeling, estimation, and control of quadrotor," IEEE Robotics and Automation Magazine, vol. 19, no. 3, pp. 20-32, 2012.

[3] J. Wang, T. Raffler, and F. Holzapfel, "Nonlinear position control approaches for quadcopters using a novel state representation," in Proceedings of the AIAA Guidance, Navigation, and Control Conference, Minneapolis, Minnesota, USA, aug 2012, pp. 1-17.

[4] K. U. Lee, Y. H. Yun, W. Chang, J. B. Park, and Y. H. Choi, "Modeling and Altitude Control of Quad-rotor UAV," in Proceedings of International Conference on Control, Automation and Systems, Kintex, Gyeonggi-do, Korea, 2011, pp. 1897-1902.

[5] H. Bouadi, S. Simoes Cunha, A. Drouin, and F. Mora-Camino, "Adaptive sliding mode control for quadrotor attitude stabilization and altitude tracking," in IEEE 12th International Symposium on Computational Intelligence and Informatics, Budapest, Hungary, 2011, pp. 449-455.

[6] H. Voos, "Nonlinear control of a quadrotor micro-uav using feedbacklinearization," in Proceedings of the IEEE International Conference on Mechatronics, Malaga, Spain, apr 2009, pp. 1-6.

[7] A. Das, K. Subbarao, and F. Lewis, "Dynamic inversion with zerodynamics stabilisation for quadrotor control," IET Control Theory and Applications, vol. 3, no. 3, pp. 303-314, 2008.

[8] A. Mokhtari and A. Benallegue, "Dynamic feedback controller of Euler angles and wind parameters estimation for a quadrotor unmanned aerial vehicle," in Proceedings of IEEE International Conference on Robotics and Automation (ICRA), New Orleans, LA, USA, apr 2004, pp. 23592366.

[9] S. Bouabdallah, "Design and control of quadrotors with application to autonomous flying," Ph.D. dissertation, Ecole polytechnique fédérale de Lausanne, 2007.

[10] M. Elsamanty, A. Khalifa, M. Fanni, A. Ramadan, and A. AboIsmail, "Methodology for identifying quadrotor parameters, attitude estimation and control," in Proceedings of the IEEE/ASME International Conference on Advanced Intelligent Mechatronics (AIM), Wollongong, Australia, jan 2013, pp. 1343-1348.

[11] L. Derafa, T. Madani, and A. Benallegue, "Dynamic modelling and experimental identification of four rotors helicopter parameters," in Proceedings of the IEEE International Conference on Industrial Technology, Mumbai, India, jan 2006, pp. 1834-1839.

[12] G. Chowdhary and R. Jategaonkar, "Aerodynamic parameter estimation from flight data applying extended and unscented Kalman filter," Aerospace Science and Technology, vol. 14, no. 2, pp. 106-117, 2010.

[13] N. Abas, A. Legowo, and R. Akmeliawati, "Parameter identification of an autonomous quadrotor," in Proceedings of the 4th International Conference on Mechatronics (ICOM), Kuala Lumpur, Malaysia, may 2011.

[14] P. Lyu, S. Bao, J. Lai, S. Liu, and Z. Chen, "A dynamic model parameter identification method for quadrotors using flight data," Proceedings of the Institution of Mechanical Engineers, Part G: Journal of Aerospace Engineering, vol. 233, no. 6, pp. 1990-2002, 2019. 

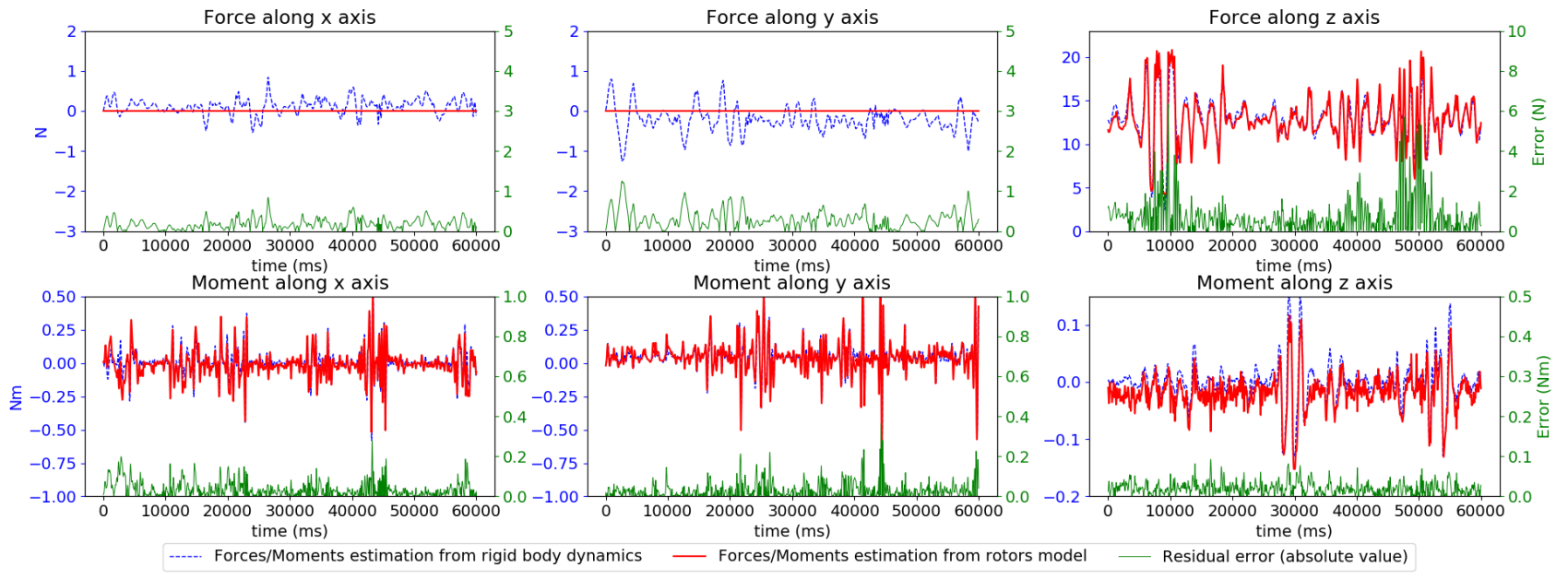

Fig. 3. Forces/Moments estimations from rigid body dynamics (blue) and rotors model (red) - Cross Validation - Configuration A - Modelling errors are represented with the scale on the right.
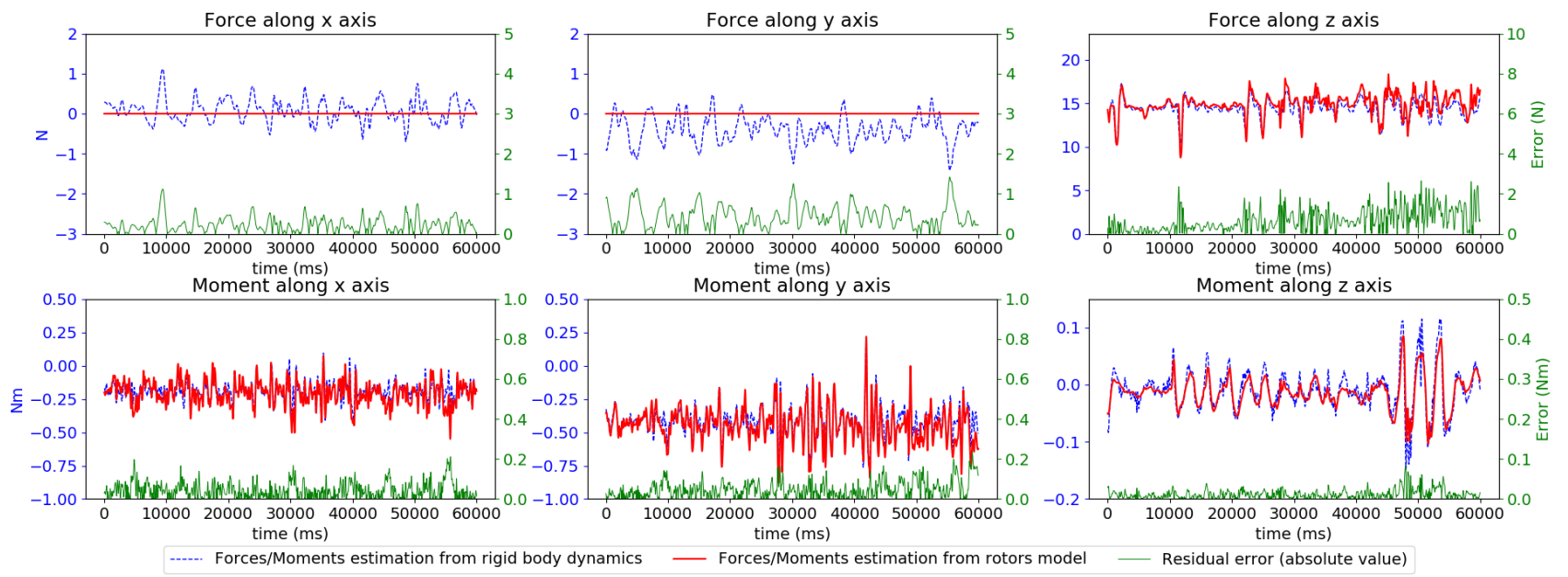

Fig. 4. Forces/Moments estimations from rigid body dynamics (blue) and rotors model (red) - Cross Validation - Configuration B - Modelling errors are represented with the scale on the right.

[15] C. Powers, D. Mellinger, A. Kushleyev, B. Kothmann, and V. Kumar, "Influence of Aerodynamics and Proximity Effects in Quadrotor Flight," in The 13th International Symposium on Experimental robotics, Québec, Canada, jun 2012, pp. 289-302.

[16] B. Theys, G. Dimitriadis, T. Andrianne, P. Hendrick, and J. De Schutter, "Wind tunnel testing of a VTOL MAV propeller in tilted operating mode," in 2014 International Conference on Unmanned Aircraft Systems (ICUAS). Orlando, FL, USA: IEEE, may 2014, pp. 1064-1072.

[17] D. Kaya and A. T. Kutay, "Modeling and Simulation of a Quadrotor using Curve Fitting Method," in AIAA Atmospheric Flight Mechanics Conference. Dallas, TX, USA: American Institute of Aeronautics and Astronautics, jun 2015.

[18] S. Sun and C. de Visser, "Aerodynamic Model Identification of a Quadrotor Subjected to Rotor Failures in the High-Speed Flight Regime," IEEE Robotics and Automation Letters, vol. 4, no. 4, pp. 3868$3875,2019$.

[19] K. Kozlowski, Modeling and Identification in Robotics. London: Springer, 1996.

[20] M. Gautier and P. Poignet, "Extended Kalman filtering and weighted least squares dynamic identification of robot," Control Engineering Practice, vol. 9, no. 12, pp. 1361-1372, 2001.

[21] W. Khalil and E. Dombre, Modeling, identification and control of robots. Kogan Page Science, 2002.

[22] S. Briot and M. Gautier, "Global identification of joint drive gains and dynamic parameters of parallel robots," Multibody System Dynamics, vol. 33 , no. 1 , pp. 3-26, 2015 .

[23] S. Sun, C. D. Visser, and Q. P. Chu, "Quadrotor Gray-Box Model
Identification from High-Speed Flight Data Quadrotor Gray-box Model Identification from High-Speed Flight Data," Journal of Aircraft, vol. 56, no. $2,2019$.

[24] J. Diebel, "Representing attitude: Euler angles, unit quaternions, and rotation vectors," Standford University, Tech. Rep., 2006.

[25] P.-J. Bristeau, P. Martin, E. Salaün, and N. Petit, "The role of propeller aerodynamics in the model of a quadrotor UAV," in Proceedings of the European Control Conference, Budapest, Hungary, aug 2009, pp. 683688.

[26] S. Bouabdallah, P. Murrieri, and R. Siegwart, "Design and control of an indoor micro quadrotor," in Proceedings of the IEEE International Conference on Robotics and Automation (ICRA), New Orleans, LA, USA, apr 2004, pp. 4393-4398.

[27] S. Van Huffel and J. Vandewalle, The total least squares problem: Computational aspects and analysis. Philadelphia, PA: SIAM, 1991.

[28] C. R. Rao and H. Toutenburg, Linear models: Least squares and alternatives, second edition. New York: Springer, 1999.

[29] J. Hollerbach, W. Khalil, and M. Gautier, "Model Identification," in Springer Handbook of Robotics, B. Siciliano and O. Khatib, Eds. Springer, 2008, ch. 14.

[30] J. Swevers, C. Ganseman, D. B. Tukel, J. de Schutter, and H. Van Brussel, "Optimal robot excitation and identification," IEEE Transactions on Robotics and Automation, vol. 13, no. 5, pp. 730-740, 1997.

[31] C. Presse and M. Gautier, "New criteria of exciting trajectories for robot identification," in Proceedings of IEEE International Conference on Robotics and Automation, Atlanta, GA, USA, 1993, pp. 907-912. 